\title{
Symmetric airfoil geometry effects on leading edge noise
}

\author{
James Gill, ${ }^{\text {a) }}$ X. Zhang, and P. Joseph \\ Aeronautics, Astronautics and Computational Engineering, Faculty of Engineering and the Environment, \\ University of Southampton, Highfield, Southampton, SO17 1BJ, United Kingdom
}

(Received 15 April 2013; revised 25 July 2013; accepted 5 August 2013)

\begin{abstract}
Computational aeroacoustic methods are applied to the modeling of noise due to interactions between gusts and the leading edge of real symmetric airfoils. Single frequency harmonic gusts are interacted with various airfoil geometries at zero angle of attack. The effects of airfoil thickness and leading edge radius on noise are investigated systematically and independently for the first time, at higher frequencies than previously used in computational methods. Increases in both leading edge radius and thickness are found to reduce the predicted noise. This noise reduction effect becomes greater with increasing frequency and Mach number. The dominant noise reduction mechanism for airfoils with real geometry is found to be related to the leading edge stagnation region. It is shown that accurate leading edge noise predictions can be made when assuming an inviscid meanflow, but that it is not valid to assume a uniform meanflow. Analytic flat plate predictions are found to over-predict the noise due to a NACA 0002 airfoil by up to $3 \mathrm{~dB}$ at high frequencies. The accuracy of analytic flat plate solutions can be expected to decrease with increasing airfoil thickness, leading edge radius, gust frequency, and Mach number. (C) 2013 Acoustical Society of America. [http://dx.doi.org/10.1121/1.4818769]
\end{abstract}

PACS number(s): 43.28.Ra, 43.28.Gq [AH]

Pages: 2669-2680

\section{INTRODUCTION}

The noise produced by interactions between an oncoming unsteady vortical gust and the leading edge of an airfoil has been of interest for many years. It is a significant contributor to, for example, the noise from wind turbines and to the noise in turbo-machinery. Additionally this noise generation mechanism has received renewed interest in relation to contra rotating open rotor engines (CRORs) which have potential to help meet Flightpath 2050 targets. The Flightpath 2050 targets call for $75 \%, 90 \%$, and $65 \%$ reductions in $\mathrm{CO}_{2}, \mathrm{NO}_{x}$, and noise emissions, respectively, against 2000 baselines. $^{1}$

A significant body of work exists on the subject of predicting leading edge gust interaction noise using analytical approaches. The early work by Sears ${ }^{2}$ (who derived a model to predict the unsteady lift and moment from a flat plate encountering a sinusoidal gust in incompressible flow) has been extended to compressible flow problems by Graham ${ }^{3}$ and Amiet. ${ }^{4}$ Amiet $^{4}$ used this theory to predict leading edge noise emissions from an isolated flat plate interacting with oncoming turbulence. Models that are used to predict leading edge noise in CROR engines (and to predict rotor-stator interaction noise in turbofans) are often still based on Amiet's flat plate model. However, the effects of real airfoil geometry on leading edge gust interaction noise have not been fully addressed, as is discussed below.

This paper applies computational aeroacoustic (CAA) methods to systematically explore the effect of real airfoil geometry on leading edge gust interaction noise. In previous studies, a variety of experimental, CAA, and analytic methods have been used to investigate the implications of assuming a flat plate geometry as opposed to modeling a realistic

\footnotetext{
a) Author to whom correspondence should be addressed. Electronic mail: j.gill@soton.ac.uk
}

airfoil shape, but these studies have not found a consensus. The results of these studies are discussed in Sec. II.

If the geometry of an airfoil is changed, then the flow surrounding the airfoil will also be altered. The steady meanflow surrounding a flat plate can be assumed to be uniform [i.e., $U(x, y)=U_{x}$, where $U(x, y)$ and $U_{x}$ are the local and freestream velocities, respectively]. For real airfoils the steady meanflow is non-uniform, but the asymptotic nature of flow features such as the leading edge stagnation region can be difficult to include in analytic models. If the implications on noise predictions are small, then complex analytical modeling of non-uniform flow may be avoided by also assuming $U(x, y)=U_{x}$ for airfoils with real geometry. The effects on noise due to differing meanflow assumptions, such as assuming a uniform flow speed throughout the flowfield, have not previously been investigated.

The current work aims to provide a better understanding of the validity of analytic flat plate models when they are used to predict leading edge gust interaction noise for real airfoils. By using a CAA code which solves the linearized Euler equations (LEEs) to describe the unsteady flow about symmetric real airfoils, this paper addresses the following:

(1) The effects of thickness and leading edge radius on the noise due to symmetric airfoils at varying Mach number, for single frequency harmonic vortical gusts convecting with a steady meanflow. The noise is studied at reduced frequencies which are higher than previously tested in computational studies.

(2) The effects on noise predictions when simplifying assumptions are made concerning the non-uniform flowfield surrounding an airfoil. The validity of assuming a uniform meanflow or an inviscid meanflow is assessed.

(3) The underlying mechanism which causes changes in the noise due to real airfoils interacting with vortical gusts, compared to predictions made with flat plate theory. 
A schematic of a single harmonic vortical gust interacting with a symmetric airfoil is shown in Fig. 1.

\section{PREVIOUS WORK}

Previous studies have investigated the effect of real airfoil geometry on the noise due to unsteady vortical disturbances impinging on an airfoil leading edge. These have included analytic, experimental, and CAA approaches, and have concentrated on the effects on noise due to airfoil thickness, angle of attack, and camber. Previous work has not considered the effects on noise due to the leading edge radius, which is an additional length-scale considered in this paper.

\section{A. Effects of angle of attack and camber}

Flat plate theory is restricted to studying the interactions between gusts and flat plates with zero camber at zero angle of attack. The effect of the angle of attack limitation on the accuracy of noise predictions has been measured by authors such as Staubs, ${ }^{5}$ Devenport, ${ }^{6}$ and Paterson. ${ }^{7}$ The effect of angle of attack on noise has been concluded to be small by all authors (reported by Devenport as approximately a $1 \mathrm{~dB}$ change between $0^{\circ}$ and $12^{\circ}$ for a NACA 0012 airfoil). In addition to considering angle of attack, Devenport also considered the effect of camber on a S831 airfoil at $30 \mathrm{~ms}^{-1}$ and found it to have a small effect on the noise, similar in magnitude to the effect of angle of attack. Because the effects of airfoil camber and angle of attack on noise predictions are expected to be small, these aspects of airfoil geometry are not considered in this paper.

\section{B. Effects of thickness}

The effect of airfoil thickness on leading edge noise has received considerable attention from previous authors, because it has a more significant effect on noise than camber or angle of attack.

\section{Experimental studies}

Paterson and Amiet ${ }^{7}$ measured the noise due to an isolated NACA 0012 airfoil interacting with nearly isotropic grid-generated turbulence at speeds of up to $165 \mathrm{~ms}^{-1}$. The
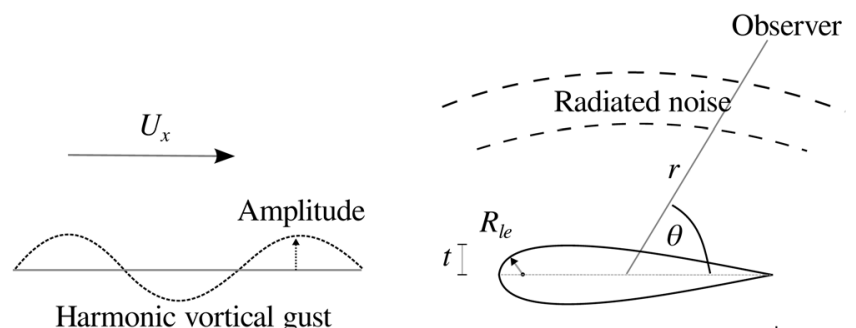

Harmonic vortical gust

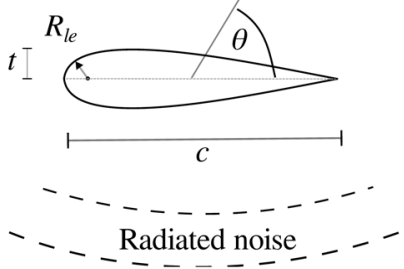

FIG. 1. A schematic of a single harmonic gust interacting with an airfoil at zero angle of attack, where $t$ is the airfoil thickness, $R_{l e}$ is the leading edge radius, $c$ is the airfoil chord, $U_{x}$ is the freestream velocity, $r$ is the observer radius, and $\theta$ is the observer angle from the downstream direction. noise spectrum and directivity were measured in addition to the surface pressure distribution on the airfoil surface. They found that the unsteady pressure on the airfoil surface was strongest near the leading edge, but a significant response was seen at all chord positions. The paper was the first to note that airfoil thickness reduces the noise compared to flat plate predictions, with the effect being more pronounced at high frequencies. A $5 \mathrm{~dB}$ reduction in noise was measured from the NACA 0012 airfoil compared with analytic flat plate solutions. However the study did not explore trends between the noise reduction and increasing airfoil thickness. The $5 \mathrm{~dB}$ reduction in noise due to the thickness of the NACA 0012 airfoil was measured at a thickness-based reduced frequency $K_{t}=t / \lambda=1$ (where $t$ is airfoil thickness and $\lambda$ is the vortical gust wavelength). This frequency was suggested by Paterson and Amiet as a measure of when flat plate theory breaks down and can no longer be considered accurate.

Another study into the effects of thickness on leading edge noise was later performed by Olsen and Wagner. ${ }^{8}$ They investigated the noise from a range of airfoils interacting with grid-generated turbulence at $94 \mathrm{~ms}^{-1}$. Airfoils with thicknesses varying from $t=0.03 c$ (i.e., 3\% thickness) to $t=0.37 c$ were used, as opposed to the single NACA 0012 airfoil considered by Amiet and Paterson. Olsen and Wagner reported that the noise reduction "increases linearly with both frequency and $t$," with thicker airfoils radiating less noise than thin ones. The apparent linear increase in the noise reduction effects with both thickness and frequency was also reported by Roger and Moreau. ${ }^{9}$ They compared measurements from several studies and found that the noise reduction effects collapsed to a single curve when thickness, flow speed, and the turbulent length scale were accounted for.

Devenport et al. ${ }^{6}$ and Hall et al. ${ }^{10}$ have both found that thicker airfoils generate less noise at high frequencies. Devenport et al. ${ }^{6}$ investigated the effect on noise of three different airfoil shapes placed in homogeneous turbulence. The three chosen airfoils had various thickness, chord, leading edge radius, and camber. Therefore the measured effects on noise for each airfoil contained influences from several length-scale changes, making it difficult to systematically study the various geometry effects on the noise. Oerlemans and Migliore ${ }^{11}$ also measured the noise from a variety of airfoil shapes placed in grid-generated turbulence. They observed a trend where airfoils with more rounded leading edges generated less noise. Hall et al. ${ }^{10}$ made changes to the front $20 \%$ of the airfoil chord by varying the leading edge thickness of an airfoil. They found that the maximum noise reduction compared to a baseline airfoil with small leading edge thickness, occurs at reduced frequencies (based on leading edge thickness) of order 1.

\section{Computational aeroacoustic studies}

Compared to experimental and analytic studies, there are fewer CAA studies which investigate the effects of airfoil geometry on leading edge noise. Furthermore, these studies have been restricted to using a small number of 
discrete frequency harmonic gusts. The use of single frequency harmonic gusts as a simplified turbulent inflow may affect the sensitivity of leading edge noise to airfoil geometry because this approach is limited to modeling gusts with parallel wavefronts. Therefore, the effects of airfoil geometry on the noise due to the variety of swept gusts that are contained in turbulent flow is not accounted for. Evers and Peake ${ }^{12}$ have shown with an analytic model to predict cascade noise that the leading edge noise due to turbulent flow exhibits a weaker sensitivity to airfoil geometry than flow containing harmonic disturbances. However, the use of harmonic disturbances is still useful in revealing general trends between leading edge noise and changes in airfoil geometry.

Atassi et al. ${ }^{13}$ numerically investigated the effects of thickness on the noise due to a harmonic gust by using a CAA method. They investigated Joukowski airfoils with thicknesses ranging from $3 \%$ to $12 \%$ of the airfoil chord. A Kirchoff method was used to predict the far-field noise based on a numerical solution of the unsteady flowfield about an isolated airfoil interacting with a periodic vortical gust. ${ }^{14}$ This study found that in $M=0.5$ flow at reduced frequencies of $K=c / \lambda \sim 1$ or higher, the effect of thickness was to reduce the noise at downstream observer locations, and increase it at upstream locations. Therefore, the basic shape of the directivity was unaltered, but the resulting pattern was skewed toward the upstream direction when compared with flat plate predictions. Atassi et al ${ }^{13}$ attributed this phenomenon to the oncoming flow "seeing a finite rounded edge at larger thicknesses (as opposed to an infinitesimally thin flat plate) from which acoustic pressure can radiate." It was also observed that the thickness effect is more pronounced at higher freestream Mach numbers.

Lockard and Morris ${ }^{15}$ performed a CAA study of noise radiated by NACA 4-series airfoils encountering harmonic vortical gusts in the time domain. They used both inviscid Euler and viscous Navier-Stokes calculations to model vortical gust interactions up to $K \sim 1.2$ in $M=0.5$ flow. Lockard and Morris made similar conclusions to Atassi et al. ${ }^{13}$ where airfoil thickness caused an upstream skewing of the directivity pattern such that the noise was reduced at downstream observer locations by a greater amount than at upstream locations. Lockard and Morris gave the realistic airfoil curvature and the realistic meanflow solution as the dominant causes for the change in the noise.

Guidati and Wagner ${ }^{16}$ used a boundary-element method to study the interaction of harmonic sinusoidal gusts with NACA 4-digit airfoils. Here, 5\%, 10\% and 15\% thick airfoils were investigated in flows with Mach number ranging from $M=0.4$ to $M=0.6$. Guidati and Wagner found again that thicker airfoils radiate less noise than thin ones, and note that the source terms in their model are highly dependent on the flow surrounding the airfoil.

\section{Analytical studies}

Most analytic models for the prediction of leading edge gust interaction noise are restricted to flat plates. However, there have been some attempts at extending the theory to include real geometry effects. By using a modified Green's function to account for plate thickness, Gershfeld ${ }^{17}$ showed that radiated sound due to turbulent flow at high frequencies could be reduced by increasing the finite thickness of a flat plate. Glegg and Devenport ${ }^{18}$ showed with a conformal mapping approach that the effect of increasing thickness is to reduce the noise at high frequencies. Moreau et al. ${ }^{19}$ modified existing flat plate analytic theory with semi-empirical corrections, based on observations of thickness effects in experimental studies. The acoustic radiation term was modified by Moreau et al. ${ }^{19}$ to account for the position of the source on a more realistic airfoil surface. Rapid distortion theory was also used to account for the distortion of small eddies by the airfoil geometry. However, the chordwise distribution of the sources was unchanged.

\section{Summary}

In the previous work described above, there is clearly some agreement between the conclusions from each study as to the effects on noise due to airfoil thickness. All studies have found that the noise is reduced at high frequency due to increasing airfoil thickness. However, there are also some contradictions between the various conclusions. Both computational works by Atassi et al. ${ }^{13}$ and Lockard and Morris ${ }^{15}$ found a forward skewing of the directivity pattern with increasing thickness. Noise measurements by Paterson and Amiet ${ }^{7}$ and Moreau et al. ${ }^{19}$ on NACA 0012 airfoils, and measurements by Olsen and Wagner ${ }^{8}$ on a range of airfoil thicknesses, did not show this behavior. Additionally, Paterson and Amiet ${ }^{7}$ suggested that flat plate theory is inaccurate for $12 \%$ thick airfoils at reduced frequencies above $K_{t}=1$ (or $K=8.3$ for $12 \%$ thick airfoils), whereas Atassi et al. ${ }^{13}$ reported that thickness effects on noise become apparent for $K>1$.

One reason why experimental measurements have not found an upstream skewing of the directivity pattern may be due to the difficulties associated with measuring acute upstream and downstream noise in a wind tunnel. The nozzle of a wind tunnel will prevent microphone placement at acute upstream angles, while the wind tunnel shear layer can affect measurements from microphones placed at acute downstream angles. Because of these factors, most experimental studies discussed here limited the range of observer angles at which noise was measured. For example, Hall et al. ${ }^{10}$ presented noise spectra at the peak radiation angle of $61^{\circ}$ from the downstream axis, Devenport et al. ${ }^{6}$ measured the noise at $90^{\circ}$, and Moreau et al. ${ }^{19}$ considered observer angles ranging between $0^{\circ}$ and $105^{\circ}$ from the downstream direction. Thus, it may be the case that previous measurements have been unable to capture the upstream behavior predicted in CAA studies. Another potential reason for the difference between conclusions of the thickness effects on noise, may be that the computational and experimental studies used differing gust inputs. The computational studies used harmonic vortical disturbances as opposed to the homogeneous turbulence generated for the experimental measurements. The use of harmonic vortical disturbances does not consider the contributions from the variety of swept gusts which are contained in a turbulent inflow, so this type of gust input may incite different leading edge noise behavior. 


\section{Mechanisms}

\section{Thickness}

It is desirable to understand the physical principles which cause the observed noise changes due to airfoil geometry, so that geometry effects on leading edge noise can be explained. Chiang and Fleeter ${ }^{20}$ found that increasing the airfoil thickness has the effect of reducing the amplitude of the leading edge surface pressure response. They also found that the location of the peak surface pressure response is moved downstream of the leading edge and is smoothed over a larger section of the airfoil chord as a result of airfoil thickness. Similar findings were reported in the analytic work of Glegg and Devenport. ${ }^{18}$ However, previous literature has not addressed why the surface pressure response is altered by the presence of airfoil thickness. This issue is addressed by the current work and is discussed in Sec. IV E.

\section{Meanflow}

Previous authors have included the accurate modeling of a non-uniform meanflow in their investigations of airfoil geometry effects on leading edge noise. For example, Lockard and Morris ${ }^{15}$ computationally studied the effects on noise due to an airfoil with thickness modeled in a nonuniform meanflow, and Evers and Peake ${ }^{12}$ also included the modeling of a realistic non-uniform meanflow in their analytical model for the prediction of airfoil leading edge cascade noise. However, it has not previously been determined if the modeling of a non-uniform meanflow is a necessary step when investigating airfoil geometry effects on noise. Analytic models are typically useful for fast repeatable noise predictions, but it can be difficult for them to include the modeling of a non-uniform meanflow. If accurate predictions can be obtained by assuming a uniform meanflow, then the complex modeling of a non-uniform flowfield can be avoided.

\section{CURRENT WORK}

This paper aims to use CAA methods to systematically study the effects on leading edge noise due to airfoil thickness $t$ and leading edge radius $R_{l e}$. Predictions will be performed of the noise due to airfoils with varying $t$ and $R_{l e}$ interacting with single frequency harmonic gusts to highlight the effects on noise due to changes in both of these lengthscale parameters. This study will also assess the validity of the flat plate assumption for modeling the leading edge noise of real airfoils. Noise predictions will be made using accurate predictions of the non-uniform meanflow around the airfoil and again when the meanflow is assumed to be uniform everywhere, in order to assess the importance of accurate representation of the non-uniform flowfield.

\section{A. Airfoil geometry definition}

For most airfoil families, such as the NACA 4-series airfoils, the leading edge radius $R_{l e}$ and the thickness $t$ are related. For example, the NACA 4-series airfoils define $R_{l e} \propto t^{2}$. If the thickness of a NACA 4-series airfoil is modified, therefore, there will be a corresponding change to $R_{l e}$. Any effects on the leading edge noise will therefore be due to a combination of these two length-scale changes. In the current work, the effects on noise of $R_{l e}$ and $t$ are investigated independently by using the NACA modified 4-series airfoil family, ${ }^{21}$ which allows separate specification of $t$ and $R_{l e}$. Leading edge radius $R_{l e}$ is related to thickness by

$$
R_{l e}=0.5\left[0.2969 \frac{t}{0.2}\left(\frac{I}{6}\right)\right]^{2}
$$

where $I$ is a non-dimensional parameter which controls the shape of the leading edge as seen in Fig. 2. $I=0$ defines an airfoil with $R_{l e}=0$, while $I=6$ represents a standard NACA 4-series profile. Through variations in $I$, the effects of leading edge radius changes with constant $t$ can be studied.

The effects on leading edge noise are studied due to gust interactions with airfoils whose thickness varies between $6 \%$ and $24 \%$, and whose $I$ parameters vary between $I=0$ and $I=10$. An additional NACA 0002 case is also included for validation of the computational method, and is the closest approximation to a flat plate that is used in the CAA method. Figure 2 shows the geometries of the airfoils investigated. The naming convention follows the standard NACA 4-series method with an additional two digits which represent the parameter $I$ and the chordwise position of maximum thickness (in tenths of chord), respectively. In this paper the chordwise position of maximum thickness position is fixed at $0.3 \mathrm{c}$ and the airfoil chord is fixed at $c=1 \mathrm{~m}$.

\section{B. Modeling methods}

The details of the analytic flat plate model and the CAA modeling strategy used in this paper are now discussed.

\section{Analytic flat plate method}

The flat plate analytic noise model due to Amiet, ${ }^{4}$ modified to allow for 2D airfoils, is used for validation of the CAA method and to assess the validity of using flat plate modeling for the prediction of real airfoil leading edge noise.

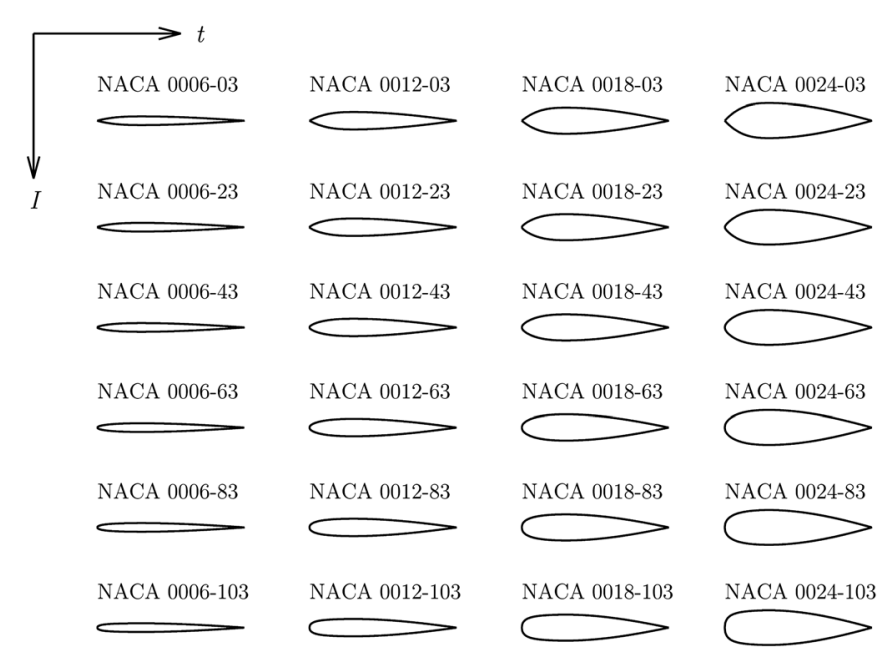

FIG. 2. The various airfoil geometries used in the study. 
The gust amplitude is taken to be constant at all frequencies, and set equal to $0.01 U_{x}$ in both the analytic and CAA methods. Gusts of this amplitude are within the linear response range that was investigated by Lockard and Morris. ${ }^{22}$

\section{CAA method}

The CAA method uses a high-order CAA code to solve the LEEs which has been used in previous aeroacoustic studies such as that by Zhang et al. ${ }^{23} \mathrm{~A}$ sixth-order compact spatial discretization scheme is used ${ }^{24}$ with tenth-order filtering and a fourth-order explicit temporal scheme. ${ }^{25}$ Unsteady gusts are superimposed onto the steady meanflow solution which convects the vortical gust toward the airfoil. The effect on noise due to different steady meanflow solutions is investigated in this paper and is discussed in Sec. IV B. Farfield noise predictions are obtained from the airfoil surface pressure response and a Ffowcs-Williams and Hawkings $(\mathrm{FW}-\mathrm{H})$ solver that is based on formulation $1 \mathrm{~A} .^{26}$

Buffer conditions are used at all edges of the simulation to prevent spurious reflections from the domain edges interfering with simulation results. An explicit damping function is utilized at the end of each timestep ${ }^{27}$ to damp perturbations to an assigned target value. The buffer zone transverse velocity target value is set equal to an unsteady value which defines the forced gust. This is done in order to prevent interactions between buffer zones and a separate gust boundary condition and is similar to the method adopted by Kim et al. $^{28}$

One-dimensional vortical gusts with velocity component normal to the freestream direction are defined in this study to be of the form of a summation of discrete frequency gusts and can expressed as

$$
w(x, T)=\sum_{i=1}^{n} w_{o} \cos \left[k_{g, i}\left(x-U_{x} T\right)\right]
$$

where $w(x, T)$ is the instantaneous gust velocity, $k_{g, i}$ is the streamwise gust wave number of the $i$ th frequency, $x$ is the streamwise location, $T$ is time, $w_{0}$ is the maximum gust amplitude (set to $w_{0}=0.01 U_{x}$ ), and $n$ is the total number of gust frequencies. Gusts at multiple discrete frequencies are defined in individual simulations, such that the vortical gust contains information at several discrete frequencies simultaneously. Sufficient numbers of frequency are chosen to resolve the spectral shape of the leading edge noise. Noise prediction at each discrete frequency is then recovered in post-processing via Fourier transformation.

C-shape grids are used near to the airfoil surface. Because of the differing grid requirements between viscous flow solutions and LEE propagation calculations, different computational grids are needed for the meanflow calculation and the gust interaction stages of the CAA method. Viscous computations are performed to obtain the steady meanflow solution where an accurate representation of the boundary layer is provided by using $Y^{+}$values of below two. For the LEE simulations the resolution requirement is to resolve the forced gust. Therefore the LEE computational grid resolution is chosen to resolve the smallest gust wavelengths by at least 12 points per wavelength. Computational grids extend to 7 chord lengths from the airfoil in all directions to prevent acoustic interference with the domain edges. To allow acoustic gusts to be overlaid onto a viscous meanflow solution, the viscous flowfield is interpolated onto the acoustic grid.

\section{RESULTS}

\section{A. Validation}

The CAA method was validated by comparing 2D analytic flat plate predictions at $M=0.2$ with noise predictions from the CAA method due to a thin NACA 0002 airfoil $(I=6, t=0.02 c)$ in a uniform meanflow. The NACA 0002 airfoil is the closest approximation to a flat plate that has been tested. An exact flat plate was not investigated with the CAA method since spatial discretization errors in finite difference codes prevent the use of genuine flat plate geometry. Lockard and Morris ${ }^{22}$ provide further discussion on the difficulties of modeling flat plates with finite difference methods. A uniform meanflow was used in the CAA prediction for better comparison with the analytic solution, which also assumes a uniform meanflow.

The leading edge far-field noise at $r=15 \mathrm{~m}$ was predicted at reduced frequencies of between $K=0.25$ and $K=12.5$. Figure 3 compares the noise predictions from analytic flat plate theory with predictions from a NACA 0002 airfoil in the CAA method, where the NACA 0002 airfoil has been modeled by assuming a uniform and a viscous nonuniform meanflow. The $\mathrm{CAA}_{\text {non-uniform }}$ spectra and directivity shown in Fig. 3 represent the CAA noise predictions in which viscous non-uniform meanflow effects around the NACA 0002 airfoil are included and are discussed in Sec. IV B.

Figure 3 shows agreement between the analytic and the $\mathrm{CAA}_{\text {uniform }}$ noise predictions of better than $1 \mathrm{~dB}$ at all tested
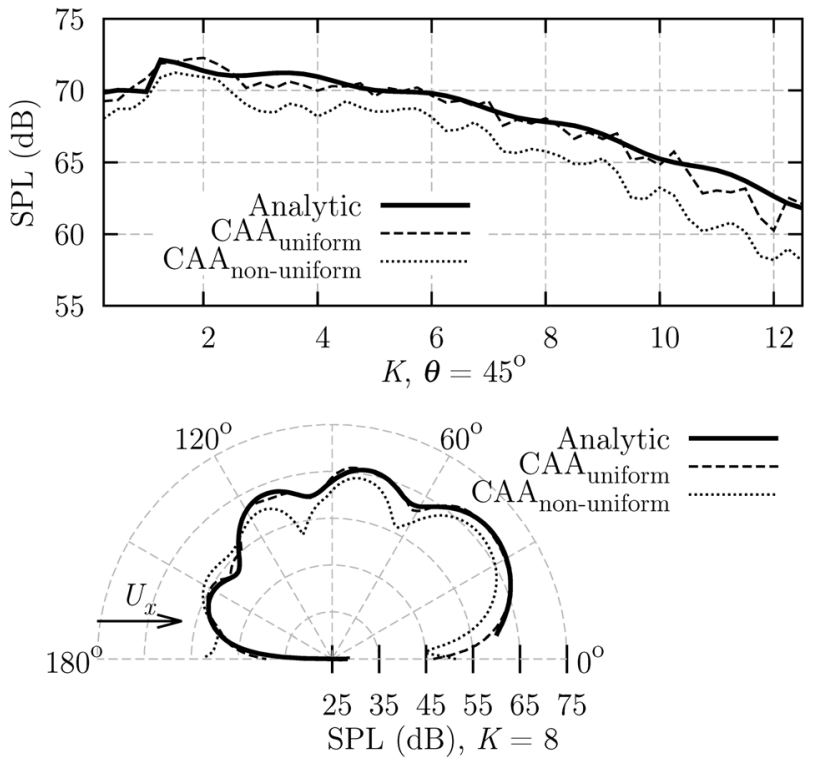

FIG. 3. (Top) Spectral and (bottom) directivity comparison of noise predictions from the CAA method using a NACA 0002 airfoil, and the analytic flat plate method. The spectral comparison is made at $\theta=45^{\circ}$ and the directivity comparison is made at $K=8$. 
gust frequencies and observer angles. The largest difference is seen in the directivity predictions at upstream observer angles, which can be attributed to the curvature of the NACA 0002 airfoil in the CAA method compared with a flat plate. Small oscillations in CAA spectral predictions can be seen. These are attributed to the assumption of discrete frequency forcing as opposed to a continuous spectrum, and to the fixed grid density, which causes each gust frequency to have a different temporal and spatial resolution in the simulation. The degree of agreement of better than $1 \mathrm{~dB}$ in Fig. 3 provides validation of the CAA methods adopted in this paper.

\section{B. Meanflow modeling assumptions}

We now investigate whether the modeling of the nonuniform meanflow is necessary for accurate noise prediction, or if accurate noise predictions can be obtained by making the assumption of uniform meanflow. This comparison also elucidates the noise generation mechanism, which is discussed in more detail in Sec. IVE. Figure 3 compares the noise predictions made with the CAA method for a NACA 0002 airfoil, by using both the viscous non-uniform meanflow solution and by assuming a meanflow that is uniform everywhere. The non-uniform meanflow case predicts a reduced noise amplitude by as much as $3 \mathrm{~dB}$ at high frequency compared to the predictions made with a uniform meanflow and also to analytic noise predictions, at all frequencies and at most observer angles. The noise difference increases slightly with increasing frequency. Therefore, even with a thin NACA 0002 airfoil, the effects of a non-uniform meanflow are important to leading edge noise predictions. Methods which do not account for the non-uniform meanflow are expected to over-predict the noise at high frequency.

Figure 4 shows the relative sound power level (PWL) predictions made using the analytic method, compared with predictions from the CAA method using a NACA 0012-63 airfoil at $M=0.2, M=0.4$, and $M=0.6$. Here, PWL is

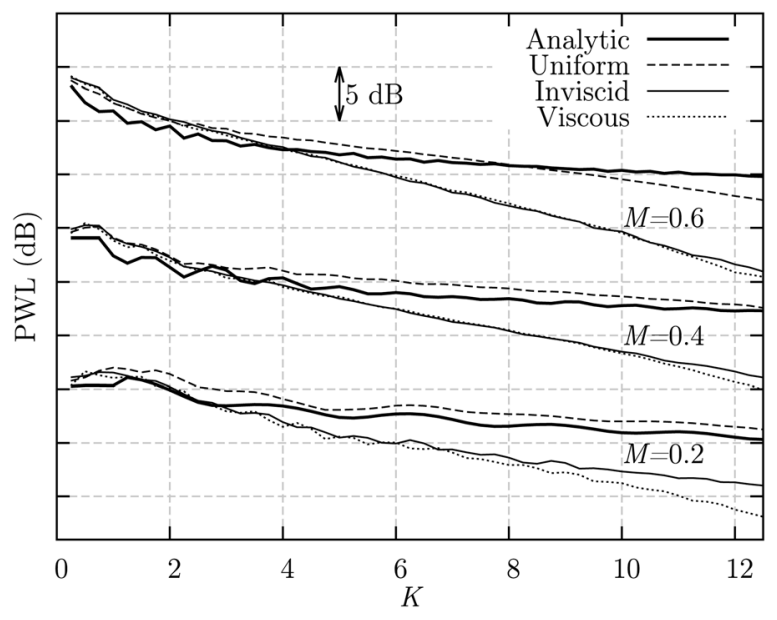

FIG. 4. PWL due to harmonic sinusoidal gusts at varying reduced frequency interacting with a NACA 0012-63 airfoil. Predictions are made at $M=0.2$, $M=0.4$, and $M=0.6$ by the analytic flat plate model and by the CAA method using differing meanflow assumptions. defined as the power per unit span for a $2 \mathrm{D}$ airfoil. ${ }^{29}$ At each Mach number, noise predictions are made by the CAA method assuming a viscous non-uniform meanflow, an inviscid non-uniform meanflow, and a uniform meanflow, so that the effects on noise due to different meanflow assumptions can be investigated.

Figure 4 shows that at all Mach numbers there is a significant difference of up to $5 \mathrm{~dB}$ between the CAA noise predictions that are made by assuming a uniform meanflow, and those made by including non-uniform meanflow effects. Predictions that include the effects of non-uniform meanflow show a reduction in noise due to the thickness of the NACA 0012-63 airfoil, whereas close agreement was obtained for the NACA 0002 airfoil in Fig. 3. This reduction increases with increasing frequency and Mach number, which is in agreement with previous literature. ${ }^{8,13}$ At $K=12$ and $M=0.6$ the CAA predictions are approximately $9 \mathrm{~dB}$ lower than the predictions made with analytic flat plate theory. However, predictions that assume a uniform meanflow show an increase in noise in most cases. Therefore the nonuniform meanflow plays an important role in the leading edge noise generation mechanism of airfoils with real geometry.

Small differences of less than $1 \mathrm{~dB}$ are seen in the noise predicted by the CAA method between using a viscous and inviscid non-uniform meanflow solution. Assuming an inviscid flowfield causes the CAA method to over-predict the noise by up to $1.5 \mathrm{~dB}$ at high frequency and low Mach number. In other parts of the spectrum the difference between the viscous and inviscid predictions is negligible. Therefore, for predictions of leading edge noise due to symmetric airfoils interacting with harmonic gusts, an inviscid flowfield can be assumed in most cases without significant loss of accuracy.

The error incurred by assuming a uniform meanflow with regards to leading edge noise prediction of real airfoils has not been previously investigated. Additionally, the small loss in prediction accuracy of up to $1.5 \mathrm{~dB}$ caused by the assumption of an inviscid flowfield has not been previously reported. The remainder of this paper sets $M=0.2$ and assumes an inviscid non-uniform meanflow. Reasons for the inaccuracy of predictions made when assuming a uniform meanflow solution are discussed in Sec. IV E.

\section{Effects of thickness}

The sensitivity of leading edge noise to varying airfoil thickness is now investigated. Figure 5 compares the contours of sound pressure level (SPL) with varying observer angle $\theta$ and gust reduced frequency $K$, between the analytic flat plate noise predictions and predictions from the CAA method using a NACA 0024-03 airfoil. Two iso-lines of constant SPL are also shown in Fig. 5 to assist comparison between the two analytic and CAA methods. A NACA 0024-03 airfoil has been chosen for the CAA prediction because it has a large thickness $t$ and zero leading edge radius $\left(R_{l e}=0\right)$, and therefore exhibits thickness effects on noise while minimizing any leading edge radius effects.

Figure 5 shows that the noise predictions from the analytic and CAA methods give similar predictions at low 


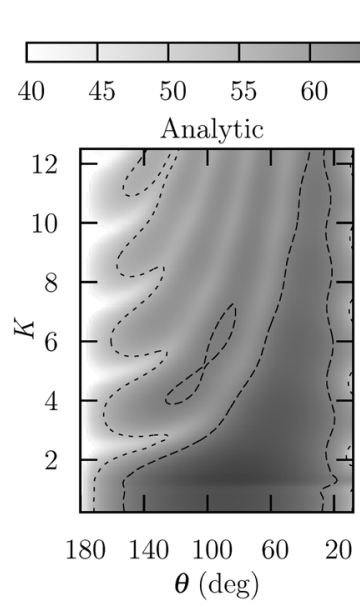

\section{SPL $(d B)$}
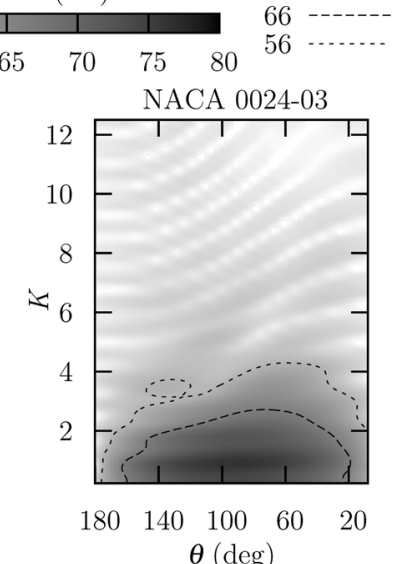

FIG. 5. Comparison between the contours of SPL from analytic flat plate predictions and the CAA method using a NACA 0024-03 airfoil, at varying observer angle and gust frequency.

frequency. However, at frequencies above $K=1$ the CAA predictions differ from the analytic predictions due to the thickness of the NACA 0024-03 airfoil. For frequencies above $K=1$ (where the gust wavelength is equal to or smaller than the airfoil chord) the effect of thickness is to decrease the noise compared to flat plate noise predictions, such that the NACA 0024-03 noise predictions are approximately $15 \mathrm{~dB}$ quieter at $K=12$. Additionally, contours of the analytic noise prediction show a strong directivity lobe at $\theta=45^{\circ}$ for $K>2$, while the NACA 0024-03 predictions exhibit less variation in noise amplitude with varying observer angle. The amplitude of the analytic flat plate noise prediction oscillates with varying frequency at forward observer angles $\left(\theta>140^{\circ}\right)$. The CAA noise prediction also shows oscillations in noise amplitude with varying frequency above $K=4$, but these oscillations are present over a wider range of angles compared with the analytical noise prediction and at different scale.

Figure 6 compares the predicted noise spectra from the analytic flat plate solution and the CAA method using a NACA 0012-03 airfoil, at two observer angles. The noise predictions from the CAA method at the downstream observer angle $\left(\theta=45^{\circ}\right)$ are lower than the analytic predictions. The difference between the two methods increases
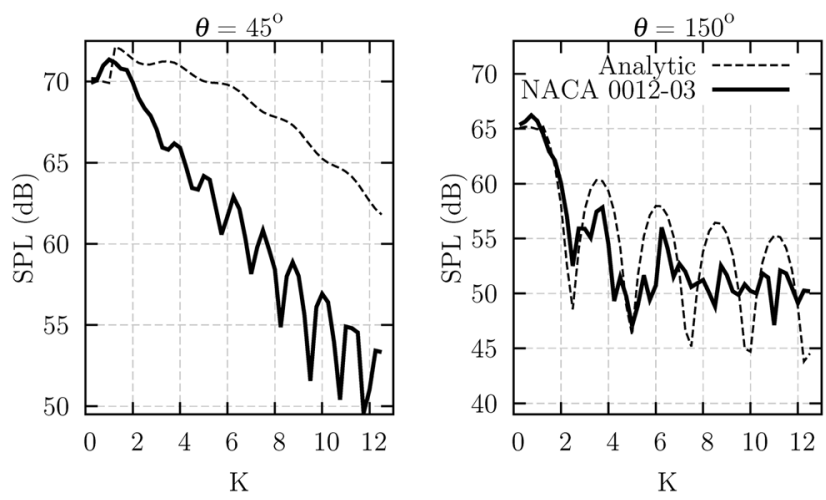

FIG. 6. Comparison of thickness effects for a NACA 0012-03 airfoil at (left) downstream observer angle $\theta=45^{\circ}$ and (right) upstream observer angle $\theta=150^{\circ}$. with increasing reduced frequency above $K=1$. This effect of thickness on the noise predictions agrees with the findings of previous authors such as Paterson and Amiet, ${ }^{7}$ who report a smaller effect than predicted here. Paterson and Amiet reported a $5 \mathrm{~dB}$ reduction in noise at $K_{t}=1$ (or $K=8.3$ for a $12 \%$ thick airfoil) due to an airfoil with $12 \%$ thickness, but Fig. 6 shows a difference of about $8 \mathrm{~dB}$. The increased sensitivity of leading edge noise to thickness, in comparison to Paterson and Amiet's work, may be due to the assumption of one-dimensional harmonic vortical gusts instead of turbulent interactions, as was reported by Evers and Peake ${ }^{12}$ in their analytical study of airfoil geometry effects on cascade noise. Figure 6 shows that for observers at $\theta=45^{\circ}$, the effect of thickness on noise is significant at $K>1$, which agrees with the conclusions of Atassi et al., ${ }^{13}$ but is lower than Paterson and Amiet $^{7}$ who conclude that flat plate theory breaks down above about $K=8.3$.

Figure 6 shows that at the upstream observer angle $\left(\theta=150^{\circ}\right)$ the thickness effects do not reduce the predicted noise in the CAA method. Here, the effect of airfoil thickness on noise is to suppress the amplitude of the oscillations that occur in the spectrum compared to flat plate predictions. This effect is significant at $K>2$ and does not vary with increasing frequency.

Figure 7 compares the directivity predictions at $K=8$ from the CAA method using airfoils with varying thickness, with those obtained from flat plate theory. Figure 7 shows that the directivity pattern remains similar as thickness is varied, but the predicted noise level reduces with increasing airfoil thickness at most observer angles. This finding agrees with the conclusions of several previous authors, including Olsen and Wagner. ${ }^{8}$ However, at acute upstream observer angles (above $\theta=130^{\circ}$ ) the noise amplitude is not reduced as a result of increasing thickness, as was also seen in Fig. 6. Lockard and Morris ${ }^{15}$ and Atassi et al. ${ }^{13}$ both reported similar behavior to that shown in Fig. 7, with the exception that both studies reported slight increases in upstream noise. This increase is not seen in the current work.

Figure 8 shows the sensitivity of the sound power $P$ prediction to airfoil thickness at constant reduced frequencies of $K=4, K=8$, and $K=12$, for airfoils with $I=0$ so that any effects on $P$ due to leading edge radius can be neglected. Solid lines are drawn as straight lines of best fit between sound power predictions and airfoil thickness. Figure 8 shows that the sound power at constant reduced frequency appears to decrease almost linearly with increasing airfoil

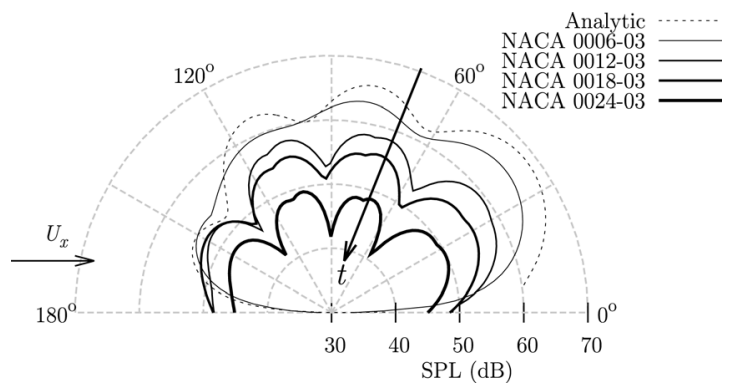

FIG. 7. The effect on noise directivity predictions due to increasing airfoil thickness, with $R_{l e}=0$, at $K=8$. 


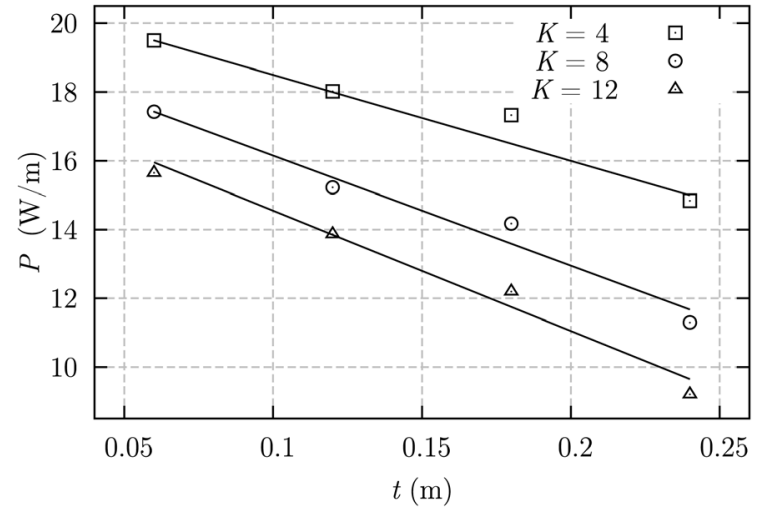

FIG. 8. The behavior of sound power $P$ with airfoil thickness at reduced frequencies of $K=4, K=8$, and $K=12$. Solid lines are drawn as lines of best fit.

thickness. The gradient, $d P / d t$, increases with increasing frequency, which is consistent with previous conclusions that airfoil thickness has a greater effect at higher frequencies. The sensitivity of sound power to thickness reported here may be consistent with the experimental findings of Olsen and Wager, ${ }^{8}$ who measured a "linear" decrease in noise due to thickness compared with the noise generated by a $3 \%$ thick airfoil. However, it is not clear if Olsen and Wagner refer to a linear change in SPL, or to a linear change in the acoustic pressure response.

\section{Effects of leading edge radius}

The effect of leading edge radius on leading edge noise is now investigated. Figure 9 shows the predicted leading edge noise spectra and directivity pattern due to a family of $12 \%$ thick airfoils with varying leading edge radii corresponding to $I=0, I=6$, and $I=10$. Figure 9 shows that the predicted noise for downstream observers is reduced by increasing the leading edge radius, and that the amount of noise reduction increases with increasing reduced frequency.
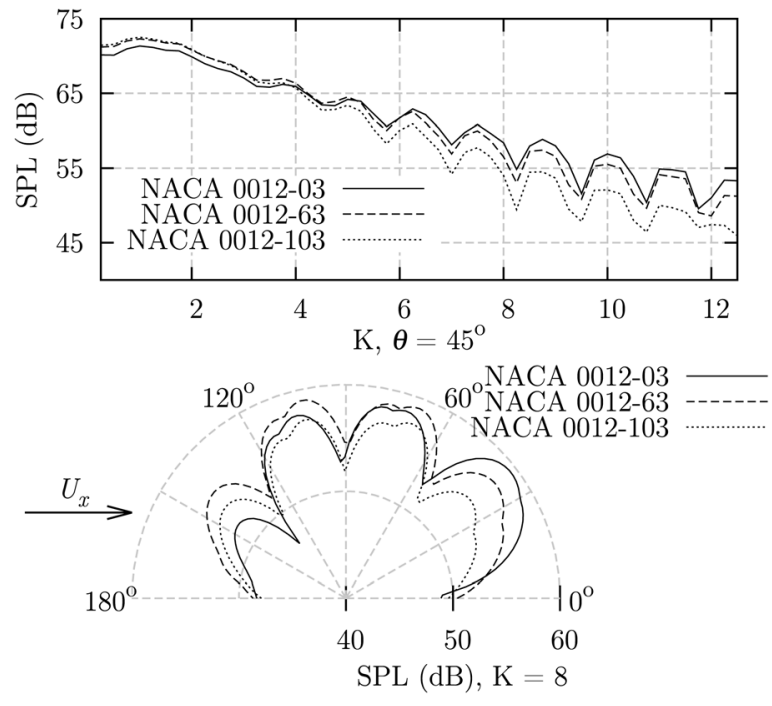

FIG. 9. (Top) Spectral and (bottom) directivity effects of the leading edge radius on noise from $12 \%$ thick airfoils. The spectral comparison is made at $\theta=45^{\circ}$, and the directivity comparison is made at $K=8$.

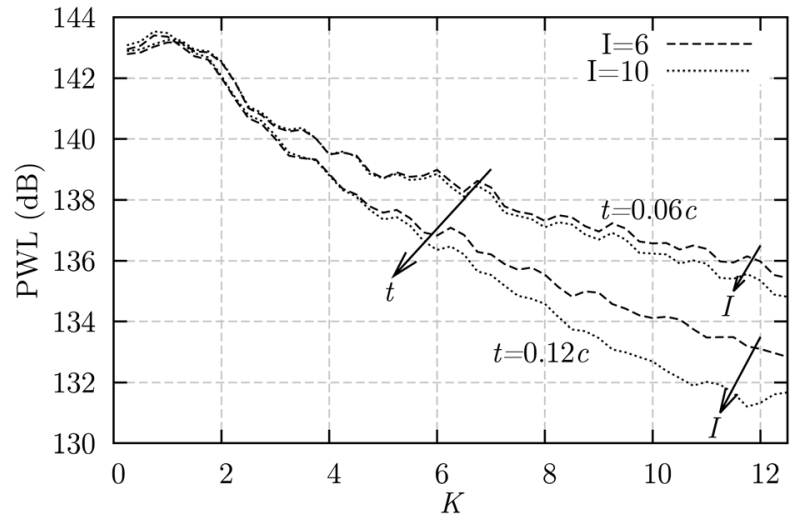

FIG. 10. Comparison of the PWL behavior with chord-based reduced frequency, for airfoils with varying thickness and leading edge radius.

At upstream observer positions there is an increase in noise due to an increase in leading edge radius, but this trend is less clear than the trend for downstream observers since noise predictions for the NACA 0012-63 airfoil are greater than for the NACA 0012-10 airfoil. In general, the effect of increasing leading edge radius is to cause a reduction in noise for downstream observers, and to cause a slight increase in noise for upstream observers. Noise predictions become sensitive to leading edge radius at reduced frequencies above about $K=4$, which is higher than the frequency of about $K=1$ at which the noise appears to become affected by airfoil thickness.

We now investigate the relative sensitivity of the effects on noise due to thickness and leading edge radius. Figure 10 shows the PWL spectra for airfoils with 6\% (or $t=0.06 c$ ) and $12 \%$ thickness, each with $I=6$ and $I=10$. At reduced frequencies above about $K=1$, the noise predictions are significantly different between airfoils with differing thicknesses, with thicker airfoils generating less noise than thinner ones. Above reduced frequencies of about $K=4$, the noise predictions for airfoils with equal thickness, but different leading edge radius, are reduced by an increase in the leading edge radius. Leading edge noise is therefore affected by airfoil thickness at lower reduced frequencies than it is affected by leading edge radius. Furthermore, the noise reduction due to the change from $6 \%$ to $12 \%$ thickness in

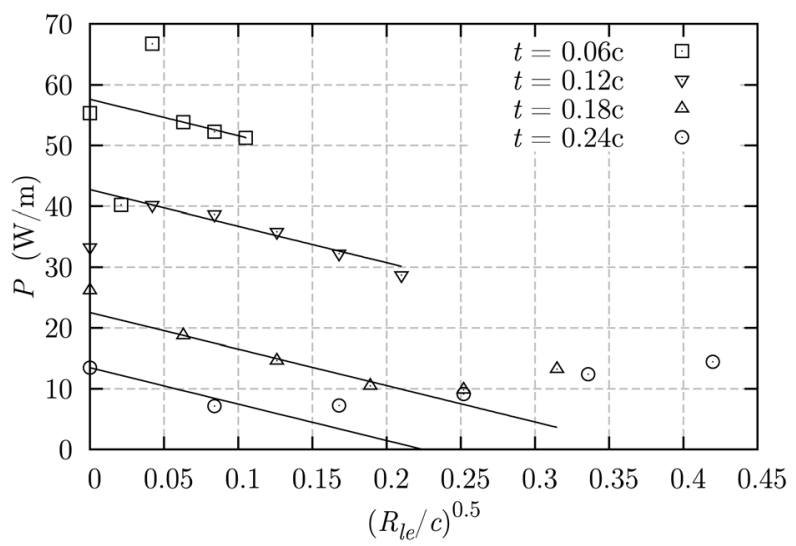

FIG. 11. The behavior of sound power $P$ with varying leading edge radius at a reduced frequency of $K=8$. Solid lines indicate linear lines of best fit. 


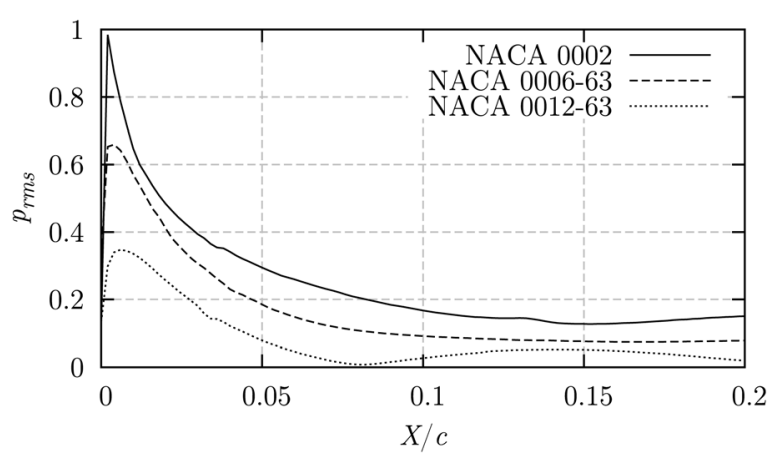

FIG. 12. Normalized airfoil surface $p_{r m s}$ response due to a vortical gust at $K=8$, for airfoils with $t=0.02 c, t=0.06 c$ and $t=0.12 c$.

Fig. 10 is about $4 \mathrm{~dB}$ at $K=12$, whereas the reduction in noise due to the change from $I=6$ to $I=10$ is about $2 \mathrm{~dB}$ at $K=12$. Therefore, leading edge noise appears to be more sensitive to the effects of airfoil thickness than to the effects of leading edge radius.

Figure 11 shows the behavior of the predicted sound power $P$ at a reduced frequency of $K=8$ with varying $\sqrt{R_{l e} / c}$. Each combination of airfoil thickness $t$ and shape parameter $I$ represents a unique value of $R_{l e}$. Each data point in Fig. 11, therefore, represents one of the 24 airfoils shown in Fig. 2. Figure 11 shows that for airfoils with constant thickness, there appears to be a linear relationship between the reduction in $P$ and increasing $\sqrt{R_{l e} / c}$ for $\sqrt{R_{l e} / c} \leq 0.2$ for this data. Some data points deviate from this apparent linear trend, particularly for airfoils with $6 \%$ thickness, but these are likely to be errors resulting from small changes in the computational grids needed to examine each separate airfoil in turn. At sufficiently high values of leading edge radius $\left(\sqrt{R_{l e} / c}>0.2\right)$ the apparent linear relationship between $P$ and $\sqrt{R_{l e} / c}$ breaks down. More extensive simulations are needed to fully identify this limit. Above the limit of $\sqrt{R_{l e} / c}=0.2$, the sound power is increased for $18 \%$ and $24 \%$ thick airfoils with increasing values of $\sqrt{R_{l e} / c}$.
However, in most applications of leading edge noise modeling such as turbo-machinery, the thickness is typically below $12 \%$ and therefore the leading edge radius of airfoil geometries is below the limit of $\sqrt{R_{l e} / c}=0.2$. Therefore, for most practical applications, an increase in the leading edge radius of an airfoil is expected to reduce leading edge noise.

\section{E. Mechanism}

This section investigates the mechanism underlying noise reductions due to increases in airfoil thickness and leading edge radius. Figure 12 shows the rms pressure $p_{\text {rms }}$ along the surface of the front $20 \%$ of the airfoil chord, due to gust interactions at a reduced frequency of $K=8$ with $2 \%$, $6 \%$, and $12 \%$ thick airfoils. Here, $p_{\text {rms }}$ values have all been normalized on the peak $p_{r m s}$ value of the NACA 0002 prediction. Figure 12 shows that as airfoil thickness is increased, the leading edge surface $p_{r m s}$ response is reduced, and also that the peak value of $p_{r m s}$ is moved downstream. This reduction in surface pressure response is the cause of the reduced far-field sound predictions in the CAA model, and agrees with the conclusions of Chiang and Fleeter ${ }^{20}$ who used an analytic method to study the effects of thickness on the surface pressure response of an oscillating airfoil. However, the previous literature is not clear why the surface pressure response is reduced for airfoils with real thickness compared to flat plate predictions. The CAA method used in this paper allows this to be investigated by visualizing the unsteady flowfield surrounding the airfoil.

Figure 13 shows a visualization of the instantaneous transverse velocity perturbations $v$ for a high frequency vortical gust with $K=8$, interacting with $2 \%, 6 \%$, and $12 \%$ thick airfoils. In this section $v$ is non-dimensionalized by the speed of sound $c_{0}$. Figure 13 shows that the gust wavefront is distorted by the real airfoil, and that this distortion increases with airfoil thickness. Distortion of the gust wavefront is caused by the velocity gradients present in the
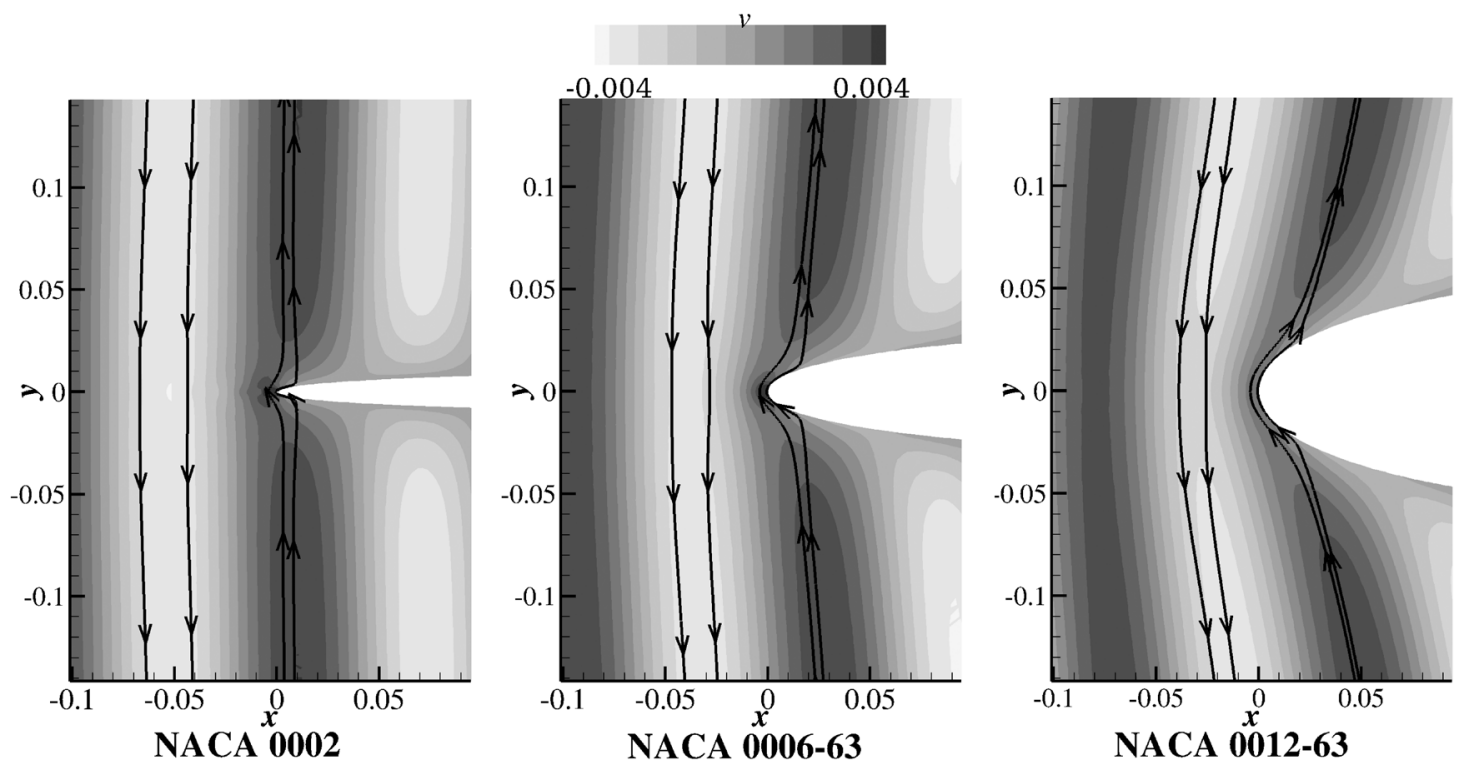

FIG. 13. Contours of $v$ for a gust at $K=8$ interacting with $2 \%, 6 \%$, and $12 \%$ thick airfoils. The contours are overlaid with streamlines to show the shape and direction of each gust wavefront. 

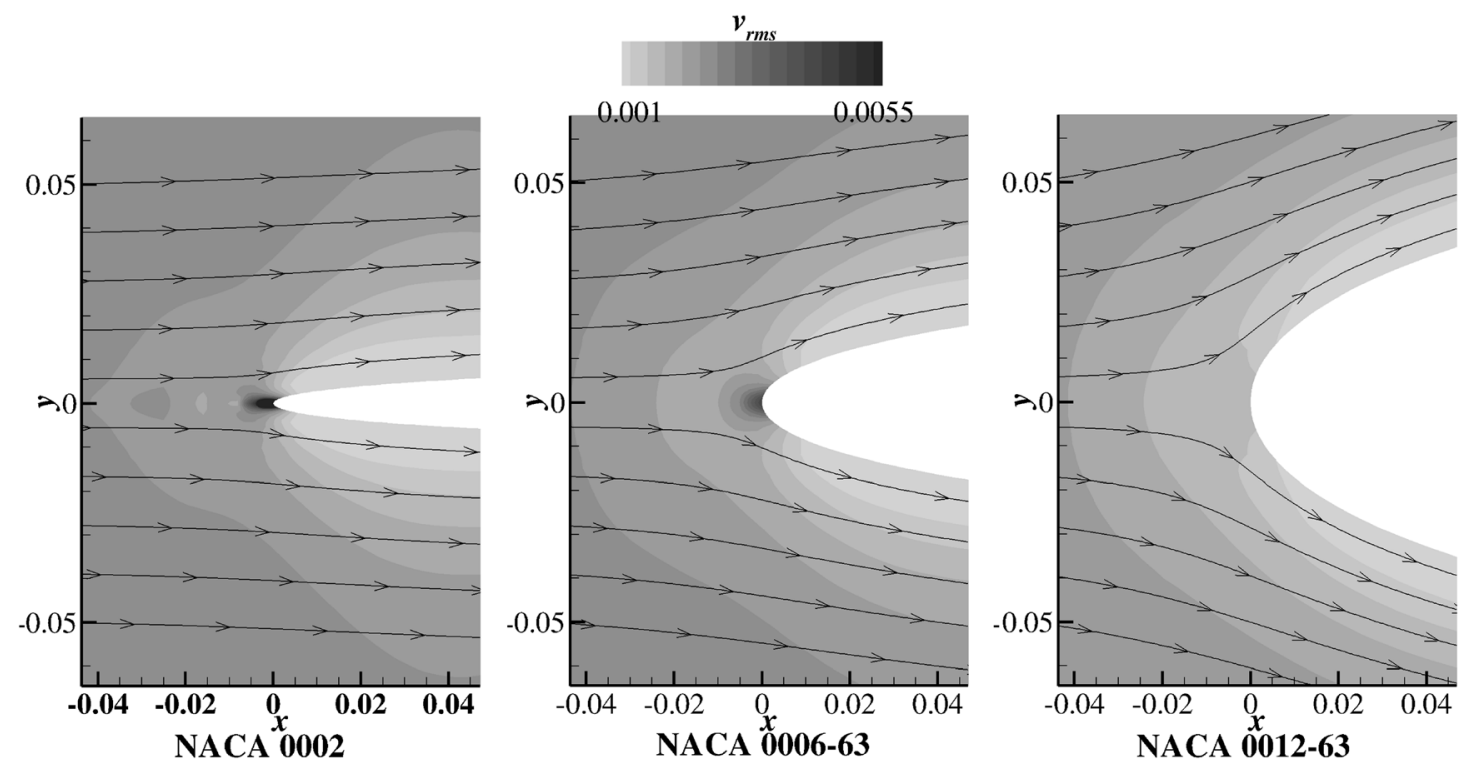

FIG. 14. Contours of $v_{r m s}$ for a gust at $K=8$ interacting with $2 \%, 6 \%$, and $12 \%$ thick airfoils. The contours are overlaid with streamlines of the non-uniform meanflow around each airfoil.

leading edge stagnation region and is stronger for airfoils with larger thickness because thick airfoils generate a larger stagnation region. Figure 13 also shows that $v$ is increased as the flow passes around the airfoil leading edge curvature. This acceleration of the flow is caused by the induced circulation around the airfoil due to the gust. However, the increase in $v$ around the leading edge is reduced when the gust wavefront is distorted by the leading edge stagnation region. Therefore, thick airfoils which cause a larger distortion of the gust wavefront will interact with reduced $v$ values at the leading edge in comparison to thin airfoils. This reduction in $v$ is therefore the main mechanism by which leading edge noise of real airfoils is reduced at high gust frequencies. The reduction in transverse perturbation velocity for thick airfoils is shown more clearly in Fig. 14, which shows contours of time-averaged transverse velocity perturbations $v_{r m s}$ over one gust cycle for three airfoil thicknesses.

Figure 15 shows contours of $v$ for a low frequency vortical gust with $K=1$, interacting with $2 \%, 6 \%$, and $12 \%$ thick airfoils. It appears that at low frequencies the airfoil thickness does not affect $v$. This is because the gust wavelength is large in comparison to the size of the stagnation region, so the gust wavefront is not significantly distorted by the velocity gradients.

In Fig. 16 contours of $v_{r m s}$ are shown for a NACA 0012-63 airfoil interacting with a vortical gust at $K=8$, when a uniform and a non-uniform meanflow is assumed in the CAA method. Figure 16 shows that when the meanflow is assumed to be uniform, $v_{r m s}$ values at the leading edge are greater in comparison to those modeled with a non-uniform
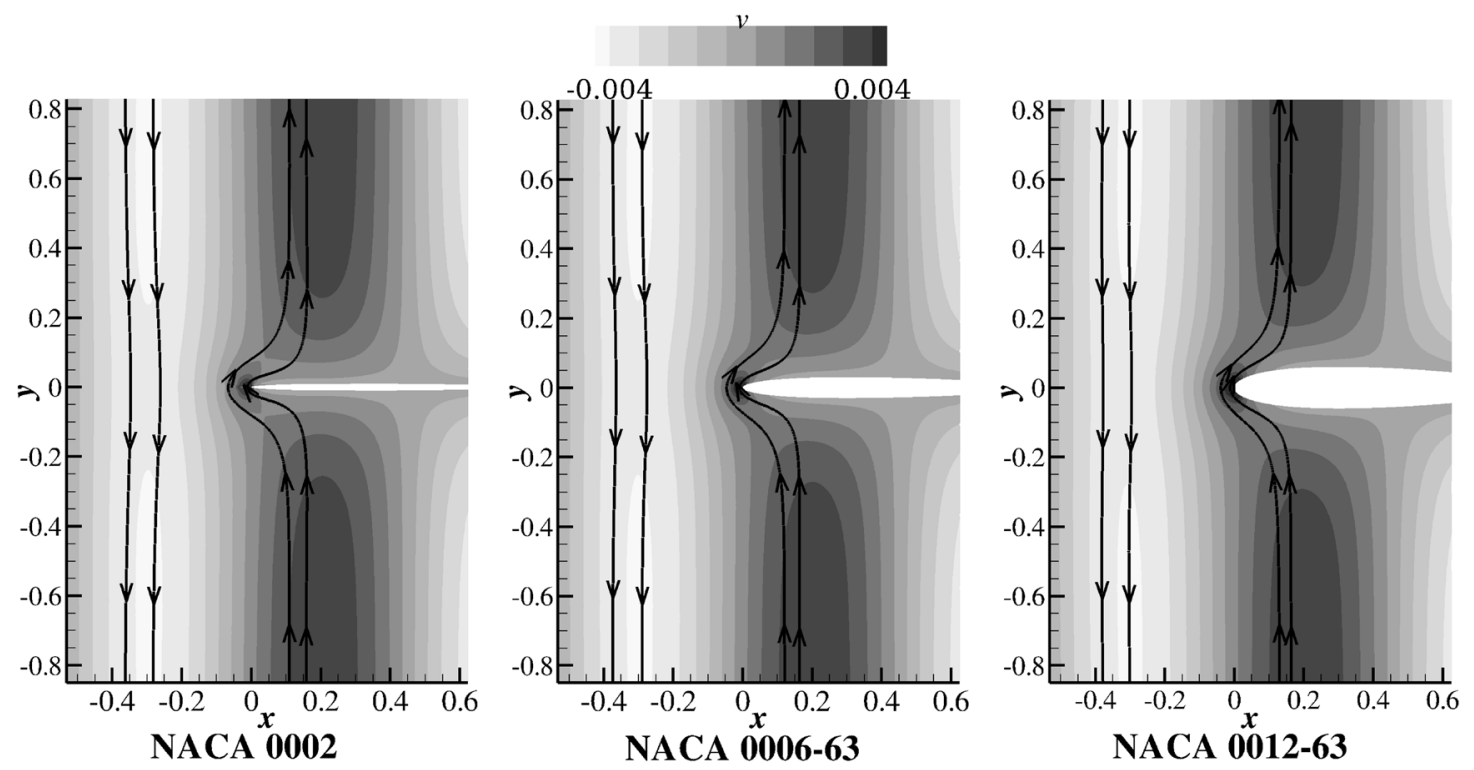

FIG. 15. Contours of $v$ for a gust at $K=1$ interacting with a NACA 0002, NACA 0006-63, and NACA 0012-63 airfoil. The contours are overlaid with streamlines to show the shape and direction of each gust wavefront. 

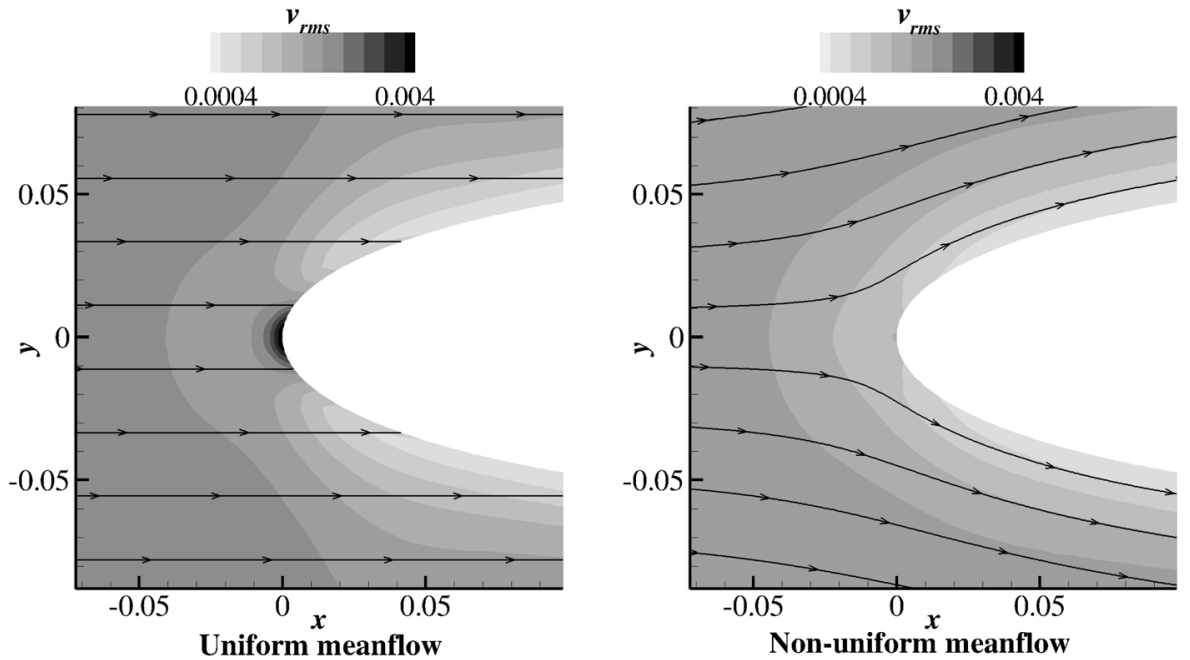

meanflow. There is no stagnation region in a uniform meanflow, so the transverse velocity reduction mechanism discussed above is not included when a uniform meanflow is assumed. This explains the inaccurate noise predictions obtained when modeling leading edge noise of real airfoils interacting with vortical gusts using a uniform meanflow.

The conclusion that the noise is affected by the leading edge stagnation region for real airfoils, implies that separating the effects on noise due to leading edge radius and to thickness is difficult. Both airfoil thickness and leading edge radius can influence the size and shape of the stagnation region because they both modify the global shape of the airfoil. Furthermore, although Figs. 13-16 indicate that the noise reduction mechanism for thick airfoils appears to be concentrated around the leading edge, the resulting reduction in surface $p_{r m s}$ for thick airfoils occurs along the entire airfoil chord. Therefore, it is the whole airfoil shape which affects the noise reduction mechanism, and the whole airfoil shape which experiences a reduced noise response. Representing a real airfoil by a single length-scale parameter, such as airfoil thickness, would ignore important aspects of the gust interaction process and would therefore produce an incorrect noise prediction.

Distortion of the gust wavefront appears to be a dominant factor in the leading edge gust interaction mechanism. This suggests that the original shape of the gust, before it is deformed, may also have an influence on the noise. This paper is limited to using one-dimensional harmonic gusts which do not vary in the transverse or spanwise directions. Therefore, a more realistic turbulent inflow that contains two- or three-dimensional disturbances may be deformed differently by the leading edge stagnation region. Further study to determine if the gust distortion and noise reduction effects are similar for twoand three-dimensional disturbances is required for a more complete understanding of the leading edge gust interaction process.

\section{v. CONCLUSIONS}

A CAA method has been applied to the modeling of leading edge noise due to harmonic vortical gusts interacting with various symmetric airfoil geometries at zero angle of attack. The effects of thickness and leading edge radius on the noise have been investigated, and the validity of flat plate analytic models has been assessed. Because the vortical disturbances have been modeled with zero transverse wave number, the analysis holds only for the interaction of an airfoil with parallel supercritical gusts. The key findings of this paper are listed as follows:

(1) The effect of increasing airfoil thickness is to reduce the leading edge noise in comparison to flat plate predictions, for reduced frequencies above about $K=c / \lambda=1$ where the gust wavelength is equal to, or smaller than, the airfoil chord. The noise is reduced in the downstream observer direction more than it is reduced in the upstream observer direction. The noise reduction effect becomes stronger with increasing frequency.

(2) The effect of increasing leading edge radius is to reduce the noise at downstream observers and to increase the noise at upstream observers, in comparison to analytic flat plate predictions. Leading edge noise becomes sensitive to leading edge radius changes for reduced frequencies above $K=4$. However, leading edge noise is less sensitive to leading edge radius than it is to airfoil thickness.

(3) Increasing the airfoil thickness $t$ causes a roughly linear decrease in the sound power due to leading edge noise at high frequencies. Additionally, increasing the square root of the leading edge radius up to $\sqrt{R_{l e} / c}=0.2$ also causes an approximately linear decrease in the sound power. The limit of $\sqrt{R_{l e} / c}=0.2$ is sufficiently high to contain most airfoil geometries which experience significant leading edge noise.

(4) The effects of airfoil geometry on leading edge noise are noticeable even for $2 \%$ thick airfoils, such that analytic flat plate predictions will over-predict the noise from a NACA 0002 airfoil by approximately $3 \mathrm{~dB}$ at high frequencies in $M=0.2$ flow. For a NACA 0012-63 airfoil this over-prediction can be up to $9 \mathrm{~dB}$ at $K=12$ and $M=0.6$. The accuracy of flat plate analytic predictions of leading edge noise can be expected to decrease with increasing airfoil thickness, gust frequency and Mach number. 
(5) The dominant mechanism that causes the discussed effects on noise is related to the leading edge stagnation region. Vortical gusts are distorted by the velocity gradients in the stagnation region such that the wavefront of the gust across the leading edge is smoothed and the gust amplitude is reduced. Because the dominant noise reduction mechanism is associated with the meanflow, It is not valid to make an assumption of uniform meanflow when modeling the leading edge noise of airfoils with real geometry. However, an inviscid meanflow can be assumed without loss of prediction accuracy in most cases.

(6) The effects on noise due to airfoil thickness and leading edge radius are linked, because both length-scales affect the overall airfoil shape which in turn affects the shape and size of the leading edge stagnation region. Rather than represent a real airfoil by a single length-scale parameter, such as airfoil thickness, the overall shape of the airfoil should be considered when modeling leading edge noise.

A limitation of the current work is that it only considers one-dimensional sinusoidal harmonic gusts at zero angle of attack. Extension of a CAA method to enable the modeling of leading edge noise via synthesis of a two- or threedimensional turbulent spectrum would provide interesting future study.

\section{ACKNOWLEDGMENTS}

The authors would like to thank the Industry Doctoral Training Center at the University of Southampton, the EPSRC, and Airbus for providing and funding the EngD project under which the current work is conducted.

${ }^{1}$ European Commission, "Flightpath 2050 Europe's vision for aviation: Report of the high level group on aviation research" (Publications Office of the European Union, Luxembourg, 2011).

${ }^{2}$ W. J. Sears, "Some aspects of non-stationary airfoil theory and its practical applications," J. Aerosp. Sci. 8, 104-108 (1941).

${ }^{3}$ J. M. Graham, "Similarity rules for thin aerofoils in non-stationary subsonic flows," J. Fluid Mech. 43, 753-766 (1970).

${ }^{4}$ R. K. Amiet, "Acoustic radiation from an airfoil in a turbulent stream," J. Sound Vib. 41, 407-420 (1975).

${ }^{5}$ J. K. Staubs, "Real airfoil effects on leading edge noise," Ph.D. thesis, Virginia Polytechnic Institute and State University, Blacksburg, VA (2008).

${ }^{6}$ W. J. Devenport, J. K. Staubs, and S. A. L. Glegg, "Sound radiation from real airfoils in turbulence," J. Sound Vib. 329, 3470-3483 (2010).

${ }^{7}$ R. W. Paterson and R. K. Amiet, "Acoustic radiation and surface pressure response of an airfoil due to incident turbulence," Technical Report CR-2733, NASA, Washington, D.C. (1976).
${ }^{8}$ W. Olsen and J. Wagner, "Effect of thickness on airfoil surface noise," AIAA J. 20, 437-439 (1981).

${ }^{9}$ M. Roger and S. Moreau, "Extensions and limitations of analytical airfoil broadband noise models," Int. J. Aeroacoustics 9, 273-305 (2010).

${ }^{10}$ A. M. Hall, O. V. Atassi, and J. Gilson, "Effects of leading-edge thickness on high-speed airfoil-turbulence interaction noise," in 17th AIAA/CEAS Aeroacoustics Conference, Portland, Oregon, AAIA Pap. 2011-2861 (2011).

${ }^{11}$ S. Oerlemans and P. Migliore, "Aeroacoustic wind tunnel tests of wind turbine airfoils," in 10th AIAA/CEAS Aeroacoustics Conference, Manchester, UK, AAIA Pap. 2004-3042 (2004).

${ }^{12}$ I. Evers and N. Peake, "On sound generation by the interaction between turbulence and a cascade of airfoils with non-uniform mean flow," J. Fluid Mech. 463, 25-52 (2002).

${ }^{13}$ H. M. Atassi, S. Subramaniam, and J. R. Scott, "Acoustic radiation from lifting airfoils in compressible subsonic flow," Technical Report AIAA90-3911, NASA, Washington, D.C. (1990).

${ }^{14}$ J. R. Scott and H. M. Atassi, "Numerical solutions of the linearized Euler equations for unsteady vortical flows around lifting airfoils," Tech. Rep. 102466, NASA, Washington, D.C. (1990).

${ }^{15}$ D. Lockard and P. Morris, "Radiated noise from airfoils in realistic mean flows," AIAA J. 36, 907-914 (1998).

${ }^{16} \mathrm{G}$. Guidati and W. Wagner, "The influence of airfoil shape on gustairfoil interaction noise in compressible flows," AIAA Pap. 99-1843 (1999).

${ }^{17}$ J. Gershfeld, "Leading edge noise from thick foils in turbulent flows," J. Acoust. Soc. Am. 116, 1416-1426 (2004).

${ }^{18}$ S. A. L. Glegg and W. J. Devenport, "Panel methods for airfoils in turbulent flow," J. Sound Vib. 329, 3709-3720 (2010).

${ }^{19}$ S. Moreau, M. Roger, and V. Jurdic, "Effects of angle of attack and airfoil shape on turbulence-interaction noise," in 11th AIAA/CEAS Aeroacoustics Conference, Monterey, California, AIAA Pap. 2005-2973 (2005).

${ }^{20}$ H. D. Chiang and S. Fleeter, "Prediction of oscillating thick cambered aerofoil aerodynamics by a locally analytic method," Int. J. Num. Methods Fluids 8, 913-931 (1988).

${ }^{21}$ C. Ladson, C. Brooks, A. Hill, and D. Sproles, "Computer program to obtain ordinates for naca airfoils," Tech. Rep. 4741, NASA, Washington, D.C. (1996).

${ }^{22}$ D. Lockard and P. Morris, "A parallel implementation of a computational aeroacoustic algorithm for airfoil noise," J. Comp. Acoust. 5, 337-353 (1997).

${ }^{23}$ X. Zhang, X. X. Chen, and P. A. Nelson, "Computation of spinning modal radiation from an unflanged duct," AIAA J. 42, 1795-1801 (2004).

${ }^{24}$ R. Hixon, "Prefactored small-stencil compact schemes," J. Comput. Phys. 165, 522-541 (2000).

${ }^{25}$ F. Hu, M. Y. Hussaini, and J. Manthey, "Low-dissipation and lowdispersion runge-kutta schemes for computational acoustics," J. Comput. Phys. 124, 177-191 (1996).

${ }^{26}$ F. Farassat and G. P. Succi, "The prediction of helicopter rotor discrete frequency noise," Vertica 7, 309-320 (1983).

${ }^{27}$ S. K. Richards, X. Zhang, X. X. Chen, and P. A. Nelson, "The evaluation of non-reflecting boundary conditions for duct acoustic computation," J. Sound Vib. 270, 539-557 (2004).

${ }^{28}$ J. W. Kim, A. S. H. Lau, and N. D. Sandham, "CAA boundary conditions for airfoil noise due to high-frequency gusts," Period. Eng. 6, 244-253 (2010).

${ }^{29} \mathrm{~V}$. Blandeau and P. F. Joseph, "Broadband noise due to rotor-wake/rotor interaction in contra-rotating open rotors," AIAA J. 48, 2674-2686 (2010). 